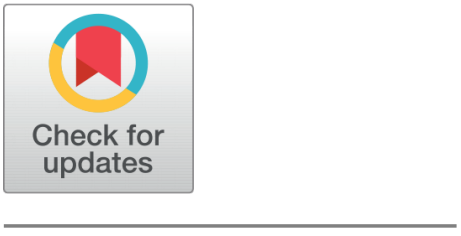

OPEN ACCESS

Received: 21.05.2020

Accepted: 30.06 .2020

Published: 03.12.2020

Citation: Shashi Raj K, Siddesh GK, Mallikarjunaswamy S, Vivek Raj K

(2020) Interference resilient stochastic prediction based dynamic resource allocation model for cognitive MANETs. Indian Journal of Science and Technology 13(41): 4332-4350. https://doi.org/ 10.17485/IJST/v13i41.687

* Corresponding author.

shashiraj18@gmail.com

Funding: None

Competing Interests: None

Copyright: (c) 2020 Shashi Raj et al. This is an open access article distributed under the terms of the Creative Commons Attribution License, which permits unrestricted use, distribution, and reproduction in any medium, provided the original author and source are credited.

Published By Indian Society for Education and Environment (iSee)

ISSN

Print: 0974-6846

Electronic: 0974-5645

\section{Interference resilient stochastic prediction based dynamic resource allocation model for cognitive MANETs}

\author{
K Shashi Raj ${ }^{1 *}$, G K Siddesh², S Mallikarjunaswamy², K Vivek Raj ${ }^{3}$ \\ 1 Department of Electronics and Communication Engineering, Dayananda Sagar College of \\ Engineering, Bengaluru, 560078, Karnataka, India \\ 2 Department of Electronics and Communication Engineering, JSS Academy of Technical \\ Education, Bengaluru, 560060, Karnataka, India \\ 3 Department of Telecommunication Engineering, Dayananda Sagar College of Engineering, \\ Bengaluru, 560078, Karnataka, India
}

\section{Abstract}

Background/Objectives: Being dynamic in nature, Mobile Ad-hoc Network (MANET) requires robust resource allocation strategy that can ensure both optimal transmission reliability and resource efficiency to meet Quality of Service (QoS) demands. The objective of this research is to address interference resilience requirement in MANETs which is must due to greedy nature of nodes especially when accessing resource or bandwidth and develop a highly robust stochastic prediction based resource allocation strategy. Methods: The proposed Interference Resilient Stochastic Prediction based Dynamic Resource Allocation model for Cognitive MANET (ISP-DRACM) intends to enable optimal resource allocation under interweave and underlay network setup with instantaneous as well as average interference conditions. It employs a joint power management and resource allocation strategy where it intends to maximize the weighted sum-rate of the secondary users under certain defined conditions like average power and stochastic interference level. Findings/Novelty: Inculcating resource allocation problem as controlled Markov Decision Process using Hidden Markov Model (HMM) and Lagrange relaxation, our proposed model achieves better resource allocation under limited noise or interference condition and hence achieves both costeffectiveness as well as QoS provision. This method has exhibited satisfactory performance towards spectrum allocation to the secondary users without imposing any significant interference for both interweave as well as underlay Cognitive Radio setup.

Keywords: Cognitive mobile ad-hoc network; stochastic prediction; interference resilience; channel state information; dynamic resource allocation; underlay and overlay cognitive MANET 


\section{Introduction}

In the last few years, the use of MANETs has increased significantly due to its adaptability towards mobile-communication, low-latency routing decision ability and operating characteristics within unlicensed spectrum bands. In major wireless communication systems, enabling resource efficiency, especially radio spectrum has always been a driving force to develop resource-efficient routing mechanism. However, the existing unlicensed spectrum is getting overcrowded day by day, thus violating QoS delivery over the network. To alleviate such issues the concept of Cognitive Radio Network (CRN) is found more viable that can enable Dynamic Spectrum Access (DSA) over dense and dynamic mobile-network conditions. CRN can play vital role to alleviate the issue of spectrum scarcity by sharing available resource amongst participating mobile nodes. CRN supports resource sharing amongst connected nodes where nodes containing both Primary User(PU)s as well as Secondary User(SU)s intend to access the underutilized resources concurrently in opportunistic manner and make spectrum heavily crowded. It makes CRN to undergo interference causing QoS violation to the PUs in MANET. It can cause uncontrollable interference causing packet drop and exceedingly high energy-exhaustion in MANETs. Hence, enabling optimal resource access and energyefficient routing is must to retain maximum data transmission with minimum interference and drop probability ${ }^{(1)}$. It can be achieved by timely spectrum sensing, resource allocation and transmission. The under-utilization of the resources (spectrum bandwidth) being one of the gaps as many users are not taking this into consideration, thus saving the energy cost and increasing the reliability. Nodes in CRNs sense the availability of licensed spectrum or allied channels with PUs and performs resource scheduling to access the idle spectrum when needed ${ }^{(1-4)}$.

However, it has always been a challenge to enable energy efficiency when sensing idle spectrum across available licensed channels and transmit certain fixed amount of data over them, which can even become more difficult for MANETs which are highly dynamic in topology. Though, a few efforts have been made to enhance QoS in CRN based WSNs, where authors have either focused on minimizing transmission delay ${ }^{(1-3)}$ or by maximizing network capacity ${ }^{(3,4)}$, not much significant effort are visible towards MANET. Authors even tried improving energy-efficiency by enhancing spectrum sensing and switching control; however, the energy-efficiency can be achieved by assuring minimum energy exhaustion for transmitting certain fixed amount of data while performing sensing and dynamic resource allocation over unlicensed channels. Practically, it is a tedious task, as it primarily depends on varied factors such as the data loss rate pertaining to the license-free channel, interference resilience to the PUs etc.

Though, a number of researches have been done towards energy-efficiency MANETs; however majority of the existing approaches either focus on reducing active sensing nodes to reduce energy exhaustion or improve routing paradigm. Under dynamic network condition, the lack of active sensing nodes might force network to undergo delayed Channel State Information (CSI) estimation or even outdated CSI estimation. The improper functional characteristics or greedy resource access nature which is common in CRNs, it might cause significantly high interference to the PUs impacting their QoS provision. A few researches have tried to enhance resource access and transmission efficiency by means of a power and channel scheduling, it could not address interference and resulting QoS violation introduced by SUs on PUs. Though, a number of researches have been done towards energy-efficiency MANETs; however majority of the existing approaches either focus on reducing active sensing nodes to reduce energy exhaustion or improve routing paradigm or could be considered as another major research gap which is imbibed as one of our objectives of the research work proposed.

Considering such limitations and gaps which are mentioned in the previous paragraph, in this study, a robust and efficient stochastic resource allocation model is developed for MANETs that intends maintaining optimal resource access to SU, while maintaining interference (caused by SU) below a specified threshold. The main advantage of the proposed work being filling up of the gaps mentioned in the previous paragraphs and proposition of new model to produce efficient results in comparison with the work done by the earlier researchers. The proposed resource allocation model is designed as a resource allocation problem as controlled Markov Decision Process using Hidden Markov Model (HMM) and Lagrange relaxation; our proposed ISP-DRACM model achieves better resource allocation under limited noise or interference condition and hence achieves both cost-effectiveness as well as QoS provision. Realizing dynamic nature of MANET and different operating environment, the proposed resource allocation has been assessed for Interweave (also called Overlay) and Underlay setup, where resource allocation has been performed under instantaneous as well as averaged interference conditions. The overall proposed model has been developed and simulated using MATLAB 2019b tool and the performance has been assessed in terms of resource allocation and power transmission scheduling.

\subsection{Previously reported work}

In ${ }^{(5)}$ proposed an energy efficient cluster head selection approach which achieves energy-efficient clustering while considering the dynamic channel access nature of CR Sensor Networks. $\operatorname{In}^{(6)}$ focused on the user association problem in cognitive 
heterogeneous networks. In ${ }^{(7)}$ focused on achieving energy efficient cooperative CRN. For network dynamism, in ${ }^{(8)}$ proposed stochastic prediction like HMM where they applied user's power and resource consumption dynamics to predict future demands for resource scheduling. In ${ }^{(9)}$ applied Bayesian and Q learning, a reinforcement learning to predict resource allocation. Undeniably, reinforcement learning has played vital role in contemporary network solutions, lack of dynamics-centric or unknown network condition, which is common in MANETs. In ${ }^{(10)}$ examined efficacy of cooperative and non-cooperative sensing methods for resource allocation. In ${ }^{(11)}$ proposed spectrum sensing and secondary data transmission rate based resource allocation. In ${ }^{(12)}$ focused on transmit power scheduling for better spectrum sensing and access control by SUs.

In ${ }^{(13)}$ proposed an iterative resource allocation concept using Dinkelbach method with Hungarian approach; though its efficiency over dynamic network like MANET remains confined. Das et al. ${ }^{(14)}$ tried to reduce SU sensing unit to minimize energy consumption. Considering interference probability in cognitive resource access or opportunistic resource access condition, in ${ }^{(15)}$ focused on energy-efficient spectrum access while assuring interference-resilience to the PUs. In ${ }^{(16)}$ developed Hybrid Radio Rendezvous (HRR) protocol for Clustered-CRN by estimating upper bound on the Maximum TTR (MTTR) to perform HRR for energy-efficient sensing; however bandwidth allocation could not be addressed. $\mathrm{In}^{(17)}$ focused on improving sensing period to reduce power consumption of SUs. In ${ }^{(18)}$ applied game-theoretic model to support PUs and SUs ability to tune their transmission power and frequencies, simultaneously. A Stackelberg game concept was designed to enable hierarchical spectrum access, though it lacked contributing efficient resource allocation paradigm. Li et al. ${ }^{(19)}$ designed a smart Relaying Opportunistic Spectrum Sharing (ROSS) model to improve energy-efficiency of both PUs and SUs.

Energy Awareness Optimal Relay Selection (EAORS) was proposed by Yang et al. ${ }^{(20)}$ using weighted objective function that reduced relay node so as to preserve energy. To achieve energy-efficiency in ${ }^{(21)}$ focused on controlling transmitting power of CR nodes. In ${ }^{(22)}$ used ratio of the spectral efficiency to the total energy consumption during signal transmission and spectrum sensing to schedule transmission. In ${ }^{(23)}$ developed ECR-MAC that enabled SUs to detect and access the unused frequency spectrum. In ${ }^{(24)}$ performed cooperative spectrum sensing and data transmission for selected SUs while maintaining minimum interference to the PUs. A similar effort was made by Kozal et al. ${ }^{(25)}$ who applied multi-hop cluster-based cooperative spectrum sensing scheme to help SU for using idle spectrum without imposing interference to the PU(s). In ${ }^{(26)}$ proposed clustered-CRN in mobile CR Ad-hoc network.

In ${ }^{(27)}$ designed a cross layer resource allocation model for multi-hop CRN under undefined transmission gain and PU's presence in network. Authors exploited channel statistics and mapping information to identify PU and its resource demand, based on which the resource allocation scheduling was performed. The authors ${ }^{(28)}$ used Bayesian model that exploiting network information to perform sensing and stochastic allocation; however could not address noise and interference caused during resource access by SU. In ${ }^{(29)}$ designed a cross-layer model based resource allocation model for CRNs. Authors at first obtained the correlation between primary receiver interference levels and energy bounds of the SUs, so as to help retaining QoS to the PUs. In ${ }^{(30)}$ focused on enhancing resource allocation while maintaining reduced transmission power and collision probability on PUs.

\section{Problem formulation}

This is the matter of fact that energy-efficiency and QoS provision (reliability, timely transmission, resource efficiency, etc) have always been the predominant need of any wireless communication systems. However, high pace rising demands of the QoS/QoE communication under diverse network condition possessing high mobility, heterogeneous network conditions and greedy resource access nature limit majority of the conventional routing approaches or protocols. On the other hand, bandwidth or resource being a constrained factor requires optimal resource-access, control and allocation strategy to meet user demands so as to continue communication. Noticeably, radio allocation has always been the challenge for industries due to non-linear demand patterns, energy-exhaustive scenario, competitive transmission nature and therefore it requires an optimal resourceallocation strategy. Undeniably, the resource allocation model must ensure both energy-efficiency as well as QoS provision to the users. Towards optimal resource utilization or allocation, in the last few years Cognitive Radio (CR) technology has gained widespread attention that intends to maximize resource utilization across the network amongst licensed users (say, PUs) and unlicensed users (say, SUs). As application specific purposes CR has given rise to a new networking paradigm where it comprises cooperatively functional PUs and SUs and SU intends to use unused resources to ensure optimal utilization.

Majority of existing works focus mainly on CR based sensor networks, which is hypothesized to be low in topological changes and network dynamism. On contrary, MANET as name reveals undergoes significantly high mobility and hence has exceedingly high non-linearity in resource utilization or demand patterns. In such case CR requires to be well versed to deal with dynamic topology and resource demands. On the other hand, CRN based MANETs requires maintaining optimal balance between sensing, resource access, transmission scheduling and allocation efficiency. Merely, reducing active nodes or MAC scheduling cannot yield optimal performance until strengthening the resource allocation policy with interference resilient-transmission 
control. In other words, scheduling resource allocation can be done better provided it allocates or schedules transmission while ensuring that the noise or interference caused by SUs on PU(s) will be significantly low so that QoS or QoE could not be affected. CRN based MANETs require maintaining energy-efficient and interference resilience to both PUs as well as SUs for QoS communication. Additionally, CR-based MANET (here onwards we call it as CRN) might function as both interweave as well as underlay which has different flexibility and operating principles towards opportunistic sensing and resource allocation to the SUs. Noticeably, in underlay CRN environment SUs are allowed to access and transmit their data opportunistically as long as it maintains interference to a defined level. On the other hand, in interweave CRN states a coexisting communication culture where SUs are required to maintain their interference level to a predefined threshold. In addition, in interweave SUs can access the spectrum which is left unused or under-utilized. However, in both these CRN environment, the transmission by SUs might impose interference to the PUs and hence can degrade QoS provision.

Considering above stated issues, in this paper we emphasized on developing a robust stochastic prediction assisted resource allocation strategy for CR based MANET or CRN under different network setup like interweave and underlay. Unlike classical researches, in the proposed resource allocation method the emphasis is made on enhancing power transmission scheduling and resource allocation while capping interference below a level. This approach is hypothesized to enable optimal resource allocation with negligible interference and hence better QoS delivery. In this paper a novel and robust Interference and Noise Resilient Stochastic Prediction based Dynamic Resource Allocation model for Cognitive MANET (ISP-DRACM) is developed to ensure optimal resource allocation under underlay as well as interweave network setup. ISP-DRACM intends to enable optimal resource allocation under interweave and underlay network setup with instantaneous as well as average interference conditions. ISP-DRACM employs a joint power management and resource allocation strategy where it intends to maximize weighted sum-rate of the SUs under certain defined conditions like average power and stochastic interference level. As probabilistic interference condition, ISP-DRACM intends to perform resource allocation under both instantaneous as well as averaged interference conditions. Inculcating resource allocation problem as controlled Markov Decision Process using HMM and Lagrange relaxation, our proposed ISP-DRACM model achieves better resource allocation under limited noise or interference condition. The proposed model has been designed for both interweave as well as underlay CRN setups. Considering working culture of both interweave and underlay CRNs, the resource allocation strategies have been examined under shortterm as well as long-term interference constraints. In CRN there can be dynamic resource demands and opportunistic resource access activity and hence the assessment of both short-term (say, instantaneous) interference as well as long-term (say, average) interference can make proposed system robust to handle or deal with any dynamic conditions.

\section{System model}

This section primarily discusses the proposed ISP-DRACM model for dynamic resource allocation in CR based MANETs. Before discussing the proposed model, a brief of CRN applied is given as follows.

Consider that the CRN under study be the MANET with unlicensed secondary user (SUs) who intends to transmit its data using available spectrum opportunistically over K spectrum or channels. We hypothesize that each spectrum or channel has the similar bandwidth and belongs to or connected to the different licensed PUs. Let the considered CR based MANET be possessing a CRN network controller (NC) especially designed to operate for gathering CSI and associated dynamic parameters to make resource and interference adaptive spectrum allocation decision, while ensuring minimum packet loss, retransmission probability, energy exhaustion and QoS violation. Functionally, NC intends to collect dynamic CSI information to make adaptive resource allocation scheduling. A snippet of the CSI model considered in ISP-DRACM is given in subsequent section.

\subsection{Channel state information estimation}

The CSI incorporated in our dynamic spectrum sensing and allocation strategy contains the details pertaining to the channel statistics. In ISP-DRACM, CSI is accessible to each node or users, where the CSI can be heterogeneous in nature, which is different for PUs and requesting SUs. It makes CSI estimation highly intricate for NC. We considered CSI heterogeneity due to two key reasons, first that the CSI accessibility for the links pertaining to PUs or SUs is different and second that the CSI can have decisive impact on resource allocation strategies. CSI for SUs (say, CR-to-CR) data transmission link can be called as static and often known (say, perfectly known). In other words, in case of CR-to-CR communication (to be noted, here CR says a participating MANET's node or sensor node requesting resource access from the PU), at each time slot, the instantaneous gain of the SUs can be obtained deterministically. Consider that at certain instant $n$ the instantaneous power gain of the channel in between the $m$ th SU transmitter and the intended receiver over the $k$ th spectrum be $h_{k, 2}^{m}[n]$. Noticeably, in $h_{k, 2}^{m}[n]$ the subscript " 2 " signifies the channel pertaining to the secondary transceiver and therefore the PUs transmitting respective data are placed at long distance from the SUs. Additionally, $h_{k, 2}^{m}[n]$ signifies the squared weight of the instantaneous fading coefficient divided 
by the noise power of the $k$-th spectrum.

In other words, $h_{k, 2}^{m}[n]$ signifies the squared magnitude of the instantaneous fading coefficient divided by the weight sum of the noise power along with the instantaneous interference caused by the $k$-th PU primary transmitter in CRN. Considering CSI of PUs in CRN it can't be often considered perfectly known, i.e., the radio-bands pertaining to the licensed users in CRN can't be sensed concurrently at each instant. Consequently, it makes CSI unknown for PUs in CRN. The CSI often used to be probabilistic in nature and varies over time. Typically, such hypotheses are viable especially when the sensing cost of the PUs is too high, such as power exhaustion in comparison to the sensing cost for the state of CR links. In ISP-DRACM, CSI information was collected by NC from PUs under different network conditions like noise level and interference. CRN operate under two predominant network setup conditions, interweave and underlay. Unlike major conventional researches where authors have merely considered conventional random interference, ISP-DRACM considers both interweave as well as underlay conditions, which provides different level of resource access environment for SUs. Realizing CRN network dynamism, we considered both perfect and imperfect primary CSI information for both interweave as well as underlay network. The brief of primary CSI estimation under interweave setup is given as follows:

\subsubsection{Primary CSI in interweave CRNS}

In interweave setup NC needs the information whether each of the radio-band is occupied or is being used. In addition, it estimates up to what extent it is being used. To achieve it, we introduced a Boolean constructs $a_{k}$ signifying the spectrum pattern of the PU in conjunction with the k-th spectrum band. Now, $a_{k}[n]$ can be the unit value (i.e., $\left.a_{k}[n]=1\right)$ only when the $\mathrm{PU}$ is active at instant $n$, else PU is stated to be idle or not using any resource. In ISP-DRACM, we applied a $2 \times 1$ matrix called belief factor, defined as (1).

$$
f_{a_{k}}[n]:=\left[\operatorname{Pr}\left\{a_{k}[n]=0\right\}, \operatorname{Pr}\left\{a_{k}[n]=1\right\}\right]^{T}
$$

The derived belief factor (1) can be hypothesized to be existing when the probability mass of $a_{k}(n]$ relies on the network history up ton instances. Being stochastic prediction based resource allocation strategy; ISP-DRACM obtains belief vector (1) in advance to make optimal resource allocation decision. Let, $s_{k}[n]$ states the Boolean variable signifying unit value (i.e., $s_{k}[n]=1$ ) when the k-th spectrum band has been sensed at $n$. On contrary, if a spectrum band $k$ is not sensed $s_{k}[n]=0$. Similarly, let $\stackrel{v}{a}{ }_{k}[n]$ be the estimated sensed outcome for $a_{k}[n]$ at $n$th instant (i.e., $s_{k}[n]=1$ ). Thus, with above conditions, we obtained CSI including the outdated CSI when $\left.s_{k}[n]=0\right)$ and probable noisy CSI due to error imposed during sensing and hence, $a_{k}[n] \neq \stackrel{\mathrm{v}}{a}_{k}[n]$.

To enable optimal and energy efficient resource allocation, it is must to avoid outdated CSI information and "gather and exploit" dynamic CSI information of $a_{k}[n]$ during CRN network lifetime. To achieve dynamic information, in ISP-DRACM model we applied the concept of controlled MDP, where we defined Transition Probability Matrix (TPM) Q possessing $(i, j)$ th activity entry. Mathematically, we define TPM as (2).

$$
Q_{i j}:=\operatorname{Pr}\left\{a_{k}[n]=i\right\} \mid \operatorname{Pr}\left\{a_{k}[n-1]=j\right\}
$$

where $\mathrm{i}, \mathrm{j}=0,1$.

Majority of the classical radio sensing methods have not considered any error probability, which can't be generalized under dynamic conditions of CR based MANETs or CRN. Hence, to alleviate the issue of the sensing error, we considered a factor called the likelihood of miss detection $P_{M D}:=\left\{a_{k}[n]=0\right\} \mid\left\{a_{k}[n]=1\right\}$. Additionally, we define a term called the likelihood of false alarm be $P_{F A}:=\left\{a_{k}[n]=1\right\} \mid\left\{a_{k}[n]=1\right\}$. Applying $P_{M D}$ and $P_{F A}$ values, we generate $2 \times 1$ matrix, given as follows:

$$
\begin{aligned}
& q_{1}:=\left[1-P_{F A}, P_{M D}\right]^{T} \text { and } \\
& q_{1}:=\left[P_{F A}, 1-P_{M D}\right]^{T}
\end{aligned}
$$

The CSI values obtained signifies the estimated states of a HMM where we have applied a Recursive Bayesian Estimation Model (RBEM) to estimate the instantaneous belief factor (1). Here, we update $f_{a_{k}}[n]$ using the following conditions.

- If $s_{k}[n]=0$, then $f_{a_{k}}[n]=Q f_{a_{k}}[n-1]$.

- If $s_{k}[n]=1$ and $\stackrel{\mathrm{v}}{a}[n]=0$, then obtain the belief vector as $f_{a_{a_{k}}}[n]:=f_{a_{k}}[n-1]$; for $\stackrel{\mathrm{v}}{a_{k}}[n]=1$, correct $f_{a_{a_{k}}}[n]$ using Bayes' rule. Here, we use (4) to update $f_{a_{k}}[n]$.

$$
\left[f_{a_{k}}[n-1]\right] l=\left(\left[q_{0}\right]_{l}\left[f_{a_{k}}[n]\right]_{l}\right) / q_{0}^{T} f_{a_{k}}[n]
$$


- If $s_{k}[n]=1$ and $\stackrel{\mathrm{v}}{a}{ }_{k}[n]=1$,

$$
\left.f_{a_{k}}[n]\right] l=\left(\left[q_{1}\right]_{l}\left[f_{a_{k}}[n]\right]_{l}\right) / q_{1}^{T} f_{a_{k}}[n]
$$

To be noted, the above derived model is similar to the RBEMs, like Kalman filter based prediction-correction. However, considering dynamic resource allocation scenario in mobile network like MANET, the conventional approaches seems confined. It becomes even more significant when the radio spectrum sensing error varies, and transition matrix $Q$ becomes unknown. Let, $\tau k$ be the time consumed during two consecutive alterations $a_{k}[n]$. In such case, to estimate $\tau k$, even the heavy-tailed distribution concept can be applied. Practically, with dynamic CRN, $a_{k}[n]$ can no longer be retained Markovian and hence (4)(5) can't be universal and therefore we defined a joint model $\left\{a_{k}[n], t_{k}[n]\right\}$, where $t_{k}[n]$ is obtained as the time consumed since the previous $a_{k}[n]$ changed. We formulated ISP-DRACM as a Markovian which has helped applying RBEM for CSI estimation and allied prediction. Obtaining the dynamic CSI information under interweave setup, we performed dynamic resource allocation, without imposing additional sensing and allied energy exhaustion conditions.

\subsubsection{Primary CSI in underlay CRN}

In case of Underlay setup, NC needs knowing the channel gains of SU to the PU channels, where it is expected that the SU might use available resource at PU, provided its interference level remains lower than a defined threshold to preserve QoS for PUs. Here, the CSI involved encompasses the values of the instant squared fading coefficient between the $m$ th SU and the $k$ th PU, divided by the noise power. We define CSI under overlay as $h_{k, 1}^{m}$, where subscript " 1 " signifies that the link contains primary receivers or PUs. For interweave CRN, $h_{k, 2}^{m}$ signifies the interference power, while the CSI over underlay $h_{k, 1}^{m}$ doesn't consider interference power. This is because we consider power of interference by PU as state variable. On contrary the same for SU is considered as design variable and with known CSI, $h_{k, 1}^{m}[n]$ can be obtained deterministically for any time instant $n$. In case of imperfections in detection or CSI $h_{k, 1}^{m}[n]$, the belief factor is derived as the composition of $\operatorname{CDF} F_{h_{k, 1}^{m}[n]}(h)$ and $\operatorname{PDF} \mathrm{v}_{k, 1}^{m}$ values. For underlay CRN, with CSI imperfections the belief vector (1) is obtained as per following approach.

Let, $s_{k}^{m}[n]=1$, when CSI $h_{k, 1}^{m}$ is sensed at $n$th time instant and $s_{k}^{m}[n]=0$, when the channel is not sensed. Considering $h_{k, 1}{ }^{m}$ to be estimated value for $h_{k, 1}^{m}[n]$, it signifies $s_{k}^{m}[n]=1$. In such case similar to the interweave CRNs, there can be two predominant kinds of imperfections; first the outdated CSI (when $s_{k}^{m}[n]=0$ ); and second noisy CSI, which could be caused due to the errors during sensing. In ISP-DRACM, we considered the time evolution of the CSI $h_{k, 1}^{m}[n]$ as Markovian possessing $q_{k}^{m}\left(h_{\text {new }}, h_{\text {old }}\right)$ as the likelihood of having $h_{k, 1}^{m}[n+1]=h_{\text {new }}$ under the condition of $h_{k, 1}^{m}[n]=h_{\text {old }}$. Again, let $f_{k}^{m}(h, n)$ be the PDF of $f_{k}^{m}(h, n)=h$, we believe that

$$
f_{k}^{m}(h, n+1)=\int_{\forall x} q_{k}^{m}(h, x) f_{k}^{m}(x, n) d x
$$

Considering sensing error issue we hypothesized to have memory less additive noise model given as (7).

$$
\mathrm{v}_{k, 1}^{m}[n]=h_{k, 1}^{m}[n]+v_{k}^{m}[n]
$$

where, the second term $v_{k}^{m}[n]$ signifies the white noise with known PDF and $f_{v_{k}^{m}(v]}$ independent of CSI $h_{k, 1}^{m}[n]$. Thus, with all noise and CSI conditions, we applied HMM that helped obtaining the belief factor $F_{h_{k, 1}^{m}[n]}(h)$ using RBEM. To implement RBEM based HMM we apply the following method:

Fors $s_{k}^{m}[n]=0$, obtaining the belief factor $F_{h_{k}^{m}[n+1]}(h)=\int_{\forall x} q_{k}^{m}(h, x) f h_{k}^{m}[n](x) d x$ and using the next instant CSI information $h_{k}(n+1]$ to alleviate sensing error, we applied Bayes' rule (8).

$$
f_{h[n+1]}(h)=\frac{f^{\mathrm{v}^{m}} h_{k}[n+1](h) f v_{k}^{m}[h-\stackrel{\mathrm{v}}{h}]}{\int_{\forall x}^{\mathrm{v}} h_{k}^{m}[n+1](x) f v_{k}^{m}(x-\stackrel{\mathrm{v}}{h}) d x}
$$

Since, the number of unobserved HMM can be indefinite the denominator of (8) can be presented as an integral. To obtain CSI under different CRN conditions, we applied different NCs, especially for PUs and SUs. Noticeably, the secondary CSI states inter-SUs or CR-to-CR (inter-node) link gains, that states the primary interference. Primary CSI is obtained either by the 
PUs activity vector alone in interweave network. On contrary, for underlay CRN, it is obtained by CR-to-PU a channel gain that doesn't consider secondary interference. Here, the secondary CSI is hypothesized to be perfectly known and hence the information pertaining to the instantaneous realization is deterministic in nature. Here, Primary CSI is stated to be uncertain, so that the belief state for the instantaneous realization could be probabilistic in nature.

\subsection{Channel state information estimation}

With the resource allocation model and allied variables be the function of $h$, the total CSI, we introduced a Boolean scheduling factor $w_{k}^{m}$ assigned with 1 only when the $m$-th SU is permitted to transmit its data over the $k$-th spectrum band, else is updated with 0 (i.e., $w_{k}^{m}=0$ ). For $w_{k}^{m}=1$, we estimated the instantaneous power transmitted by $m$-th SU over $k$ th channel as $p_{k}^{m}$. Considering QoS centric and reliable transmission with Bit Error Rate (BER) constraints, we coupled $p_{k}^{m}$ as well as instantaneous rate and estimate a "Rate-Power Coupling Function (RPCF) $C_{k}^{m}\left(h_{k, 2}^{m}, p_{k}^{m}\right)$. Noticeably, we assume that $C_{k}^{m}\left(h_{k, 2}^{m}, p_{k}^{m}\right)$ can be presented using Shannon's capacity formula $\log \left(1 h_{k, 2}^{m}, \frac{2 p_{k}^{m}}{k_{k}^{m}}\right)$, where $k_{k}^{m}$ signifies SNR-difference. For those systems applying Adaptive Modulation and Coding (AMC) for power transmission scheduling, the formula can be replaced with a piecewise linear function using the rates obtained through AMC modes. The SU or allied network functions in block-by-block approach, where the duration of each block signifies the coherence time of the respective fading channel. In this manner, NC estimates CSI per slot $h$ and obtains the value of $w_{k}^{m}$ and $p_{k}^{m}$. Being time-dependent CSI $h$ to varies per slot and therefore $\left\{w_{k}^{m}, p_{k}^{m}\right\}$ as well depend on CSI $h$. Thus, $\left\{w_{k}^{m}, p_{k}^{m}\right\}$ too vary over time. In ISP-DRACM, $w_{k}^{m}(h)$ and $p_{k}^{m}(h)$ has been replaced by $[n], w_{k}^{m}[n]$, and $p_{k}^{m}[n]$, to predict resource allocation stochastically. To implement it over MANET, we focus on employing adaptive resource allocation leveraging the instantaneous secondary CSI and the uncertain primary CSI. It is accomplished by identifying which SU must be transmitting data per band, and with what rate and permissible interference (power) level. Unlike classical methods, we intend to optimize resource allocation to the SU in reference to the dynamic network conditions like interference, power etc.

\subsection{Resource allocation in CRN: An optimal reference model}

Unlike classical resource allocation models, in ISP-DRACM we intended to apply different constructs like network dynamism, network parameters under different interference conditions, CSI information etc. that eventually strengthens it to enable QoS centric and power efficient resource allocation strategy. To achieve it, we formulated overall resource allocation problem by identifying optimal variables, optimization metrics and operations conditions or constrains to be meet. We considered $\left\{w_{k}^{m}, p_{k}^{m}\right\}$ as optimization variable while the weighted sum-average rate (9), is considered as optimization metrics.

$$
\bar{C}:=\sum_{k, m} E_{h}\left[\beta^{m} w_{k}^{m}(h) C_{k}^{m}\left(h_{k, 2}^{m}, p_{k}^{m}(h)\right)\right]
$$

In (9), $E_{h}$ signifies expectation throughout the CSI realizations, while $\beta^{m}>0$ presents a priority factor that depends on user's specific needs. Noticeably, the priority factor helps scheduling resources to the requesting SUs. In ISP-DRACM, we hypothesize that only the rate of SU's channel pair with $w_{k}^{m}(h)=1$ can perform resource access and transmission. To perform optimization, $p_{k}^{m}$ must always be non-negative, while $w_{k}^{m}$ belong to the set $\{0,1\}$. A SU can transmit over each band $\mathrm{k}$, provided it fulfills the following condition.

$$
\sum_{k} w_{k}^{m}(h) \leq 1, \quad \forall k
$$

A SU fulfilling the condition $\sum_{k} w_{k}^{m}(h)=1$, only can access the resource else can't access it. This is because violating above equation might impose interference and can impact QoS provision to the PUs. Under such condition, we schedule SU to transmit data at the highest average (log-term) power of (11).

$$
E_{h}\left[\sum_{k} w_{k}^{m}(h) p_{k}^{m}(h)\right] \leq \stackrel{\mathrm{v}}{p}^{m}, \forall m
$$

Under such circumstances the optimal resource allocation can be performed by achieving the solution for the problem mentioned in (12).

$$
\overline{C^{*}}:=\max _{w_{k}^{m}(h) p_{k}^{m}(h)} \sum_{k, m} E_{h}\left[\beta^{m} w_{k}^{m}(h) C_{k}^{m}\left(h_{k, 2}^{m}, p_{k}^{m}(h)\right)\right]
$$

Before discussing the resource allocation under interference a brief of the optimal resource allocation without interference is given in the sub-sequent section. 


\subsection{Resource allocation in CRN}

Before discussing resource allocation problem under interference, a brief of the resource allocation model without interference is discussed in this section. As depicted in (12), even if it depicts a non-convexity problem, it can be solved or relaxed to an equivalent convex problem using Karush-Kuhn-Tucker (KKT) conditions. The derived model (12) signifies a weighted sumrate optimization problem for a channel, where $\pi^{m}$ states the Lagrange relaxation parameter which is associated to the key parameters or constraints as derived in (11). Thus, the solution for this problem can be give as follows:

$$
\begin{gathered}
\varphi_{k}^{m}\left(p_{k}^{m}[n]\right):=\beta^{m} C_{k}^{m}\left(h_{k, 2}^{m}[n], p_{k}^{m}[n]\right)-\pi^{m}[n] p_{k}^{m}[n] \\
p_{k}^{m *}[n]:=\left[\underset{p_{k}^{m}(n]}{\operatorname{argmax}} \varphi_{k}^{m}\left(p_{k}^{m}[n]\right)\right]_{0}^{\infty} \\
=\left[\frac{\beta^{m}}{\pi_{m}[n]}-\frac{p_{k}^{m}}{k_{k, 2}^{m}}\right]_{0}^{\infty} \\
w_{k}^{m *}[n]:=1\left\{\underset{\left\{\left(m=\operatorname{argmax} \varphi_{k}^{l}\left(\left(p_{k}^{l *}[n]\right)\right) \varphi_{k}^{m}\left(p_{k}^{m *}[n]\right)>0\right\}\right.}{ }\right.
\end{gathered}
$$

Observing above expressions, it can be found that $\varphi_{k}^{m}($.$) as derived in (13) can be vital to solve or provide solution for (12).$ Here, we consider (13) as a factor signifying user's "Quality" where the transmission rate is considered as "Reward". Noticeably, in ISP-DRACM cost signifies transmission power where $\beta^{m}$ and $\pi_{m}[n]$ would be corresponding prices. Here, $\varphi_{k}^{m}(x)$ states the results of the Lagrange relaxation for (12) where the transmission power is $p_{k}^{m}[n]=\mathrm{x}$ with $w_{k}^{m}[n]=1$. Based on the estimated value of $\varphi_{k}^{m}\left(p_{k}^{m}[n]\right)$, (14) which signifies that the power $p_{k}^{m *}[n]$ is estimated for each channel (SU-PU-pair) distinctly. Model in (16) states that with the suitable or optimal scheduling variables $\left\{w_{k}^{m *}[n]\right\}_{m=1}^{M}$ per channel $k$, we don't require additional information except $k$. Considering logarithmic rate-power function, we used classical water filling algorithm for power flow analysis (15). The model derived (16) states the resource per user scheduling is opportunistic, which can be greedy in nature as well, especially under CRN condition. It reveals that only those SUs possessing the highest "Quality" must be scheduled for spectrum access. Even though $\pi^{m}[n]$ be constant and assigned as $\pi^{m *}$, it can be optimized to solve duality-problem with (12), where stochastic model can be applied to solve it. In our proposed ISP-DRACM model, we applied HMM with Lagrange relaxation to achieve optimal values of the optimization matrix to achieve interference resilient resource allocation to the users. The detailed discussion of the proposed ISP-DRACM model for resource allocation under different CRN setups and allied interference constraints is given in the subsequent sections.

\subsubsection{Resource allocation under interference conditions}

Observing literatures, it can be found that though numerous efforts have been made towards resource allocation; however majority of the researches focus on CRN without interference and noise condition, which cannot be optimal in practical MANET scenario. Considering this fact, we have focused on performing resource allocation under different noise and interference conditions. Here, we assume that limiting the average interference power and noise can help making optimal (interference-resilient) resource allocation. To ensure QoS to the licensed users or PUs in CR based MANET, we focused on confining the interference caused by SUs. Towards this motive, identifying and suppressing the probabilistic constraints can help reducing CSI imperfections that eventually will make resource allocation efficient. Interference cancellation and allied resource allocation can be achieved under two distinct methods, short term interference or long term interference. These interference models are also called as instantaneous and average interference, respectively. Here, instantaneous constraints require maintaining a defined interference probability at each instant. On contrary, the average interference constraints enable PUs to be interfered up to a tolerable level over certain duration. Functionally, instantaneous constraints are more restrictive than the average interference constraint. Thus, for $\mathrm{CRN}$ a SU can expect transmitting higher data rate under average interference constraints. Interestingly, resource allocation optimization is relatively easier in case of instantaneous constraints, while the same can't be easy for average interference constraints and hence it requires dual (optimization) scheme to solve it. In addition, there are different interference conditions such as underlay and interweave that introduce interference distinctly in CRN settings. 
Here, we have defined the duality problem for resource allocation under underlay CRN setting. Unlike existing methods, in ISP-DRACM we considered different interference conditions such as interweave and underlay under instantaneous and average constraints condition to perform resource allocation. Here, our prime motive is to design a robust dynamic resource allocation model which can be applied in any operating MANET conditions without imposing computational overheads and energy exhaustion. The details of the resource allocation for the instantaneous and average constraints is given in the sub-sequent sections.

\subsubsection{Instantaneous interference constraints}

Considering QoS provision to the PUs in CRN, it is inevitable to maintain and control the interference caused by SUs. To achieve it under instantaneous interference condition (also called short-term interference), we applied a threshold level called the maximum Interference probabilityo $k \in(0,1)$, which is assigned to the radio-band. In case of instantaneous interference constraints, in a channel $\mathrm{SU}(\mathrm{s})$ are expected to maintain interference and noise to a predefined level to ensure QoS provision. In ISP-DRACM, we performed resource allocation under instantaneous interference with both interweave setup as well as underlay. A snippet of the methods applied is given as follows:

\subsubsection{Interweave CRN}

. For interview CRN, interference might come into existence when $a_{k}[n]=1$ and $\sum_{m} w_{k}^{m}=1$, signifying that the SU can transmit data over $k-$ th channel. In such case, the interference probability can be obtained as (17).

$$
\operatorname{Pr}\left\{a_{k}[n] \sum_{m} w_{k}^{m}[n]=1 \mid n\right\} \leq \stackrel{\mathrm{v}}{\mathrm{o} k} \forall n
$$

In (11), at time slot $n, a_{k}[n]$ is measured randomly. Thus, it can be reframed as (18).

$$
E_{a_{k}[n]}\left[1_{\left\{a_{k}[n] \sum_{m} w_{k}^{m}[n]=1\right\}}\right] \leq \stackrel{\mathrm{v}}{\mathrm{o} k}
$$

Hypothesizing $\sum_{m} w_{k}^{m}[n]$ to be a deterministically known Boolean, we can derive (18) as (19).

$$
E_{a_{k}(n]}\left[1\left\{a_{k}(n]=1 \sum_{m} w_{k}^{m}(n]\right\}\right] \leq \stackrel{\mathrm{v}}{o k}
$$

Being related to the belief factor $a_{k}[n]_{2}$, we derive it as (20).

$$
\sum_{m} w_{k}^{m}[n]=1 \text { only if } f a_{k}[n]_{2} \leq \stackrel{v}{o} k
$$

To meet the interference condition under interweave set up the optimal resource scheduling can be achieved as per (21).

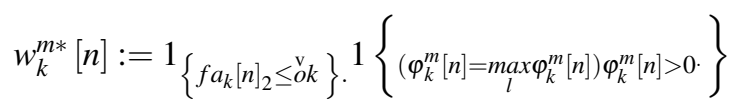

Summarily, a SU can access the channel provided it maintains the likelihood of the spectrum accessed or to be used lower than $\stackrel{\mathrm{v}}{o} k$. In case of noisy and outdated CSI, the probability primarily depends on the past CSI and allied sensing accuracy. For known and perfect CSI, the belief factor $f a_{k}[n]_{2}$ can either be zero or one, and therefore the SU can perform transmission only when the spectrum is idle. In other words,

$$
w_{k}^{m *}[n]:=1_{\left(a_{k}(n]=0\right\} \cdot 1}\left\{\left(\varphi_{k}^{m}[n]=\max _{l} \varphi_{k}^{m}[n]\right)^{\left(\varphi_{k}^{m}[n]\right.}>0\right\}
$$

\subsubsection{Underlay network}

. For underlay CRN, interference comes into existence when a PU finds received interference power caused due to SU's transmission higher than a threshold $\Gamma_{k}$. In such case, it follows the condition $w_{k}^{m}[n]>0$ and $p_{k}^{m}[n] h_{k, 1}^{m}[n]>\Gamma_{k}$. Under such circumstances, to enable optimal resource allocation, we follow the following condition for each time-step.

$$
\operatorname{Pr}\left\{p_{k}^{m}[n] h_{k, 1}^{m}[n]>\Gamma_{k} \mid n\right\} \leq \stackrel{\mathrm{v}}{o} k
$$

At $n, h_{k, 1}^{m}$ being random gives rise to the constraint (24). 
$E_{h_{k, 1}^{m}[n]}\left[1_{p_{k}^{m}[n] h_{k, 1}^{m}[n]>\Gamma_{k}}\right] \leq \stackrel{\mathrm{v}}{o} k$

And hence,

$$
E_{h_{k, 1}^{m}[n]}\left[1_{\left\{h_{k, 1}^{m}[n]<\Gamma_{k} /[n]>\Gamma_{k} p_{k}^{m}[n]\right\}}\right] \geq 1-\stackrel{\mathrm{v}}{o k}
$$

Applying the derived belief factor for primary CSI at $n$, we find

$$
f h_{k, 1}^{m}[n]\left[\frac{\Gamma_{k}}{p_{k}^{m}[n]}\right]>1-\stackrel{v}{o k}
$$

With $p_{k}^{m^{\prime}}[n]$ as the root of $\stackrel{v}{o} k=F h_{k, 1}^{m}[n] p_{k}^{m^{\prime}}[n] / \Gamma_{k}$, it signifies $p_{k}^{m}[n] \leq p_{k}^{m^{\prime}}[n]$ and therefore the interference constraint is depicted in the form of the maximum power constraint. It exhibits a vital significance as the constraint stated in (24) signifies non-convexity and hence the maximum power constraint can be convex. It avoids any multiplier to enforce the constraint and maintains the same Lagrange (multiplier) and the value of $\varphi_{k}^{m}\left(p_{k}^{m}[n]\right)$ remains same as without interference condition (13). Moreover, the scheduling can't be vital in moulding interference to meet the condition for $w_{k}^{m *}[n]$ in (16). Additionally, the optimum power (14) needed to be updated to fulfill the condition $p_{k}^{m}[n] \leq p_{k}^{m^{\prime}}[n]$. To deal with this problem, we applied a scalar projection method that gives rise to (25).

$$
p_{k}^{m *}[n]:=\left[\underset{p_{k}^{m}(n]}{\operatorname{argmax}} \varphi_{k}^{m}\left(p_{k}^{m}[n]\right)\right]_{0}^{p_{k}^{m^{\prime}}[n]}
$$

Noticeably, with the perfect CSI, as there is no uncertainty in $h_{k, 1}^{m}[n]$ we schedule the upper limit of the SU's power transmission as $p_{k}^{m^{\prime}}[n]:=h_{k, 1}^{m}[n] / \Gamma_{k}$ that as a result ensure no interference on PUs in CRN.

So far we discussed the interference-resilient resource allocation in CR-based MANET under short-term interference conditions or instantaneous interference conditions. However, realizing the long-term interference which can be possible due to pre-established CRN encompassing multiple cooperatively functional cognitive (MANET) nodes, we have developed resource scheduling strategy to handle aforesaid issue. The detailed discussion of the proposed resource allocation model under longterm interference constraint is given in the sub-sequent section.

\section{A. Long-term interference under interweave CRN setup statistical analysis}

In practice, for interweave $\mathrm{CRN}$ the main problem is not in satisfying the interference constraint, but in retrieving the likelihood of a PU to be active. Though, it is possible by applying efficient sensing approaches. In case of long-term interference PUs are expected to be in under interweave setup. We used a dual relaxation method to perform interference-resilient resource allocation. For instantaneous interference condition interweave setting needs fulfilling the condition (27).

$$
\operatorname{Pr}\left\{\Sigma_{m} w_{k}^{m}[n] a_{k}[n]=1 \mid n\right\} \leq \stackrel{\mathrm{v}}{o} \text { Or } \text { Or } \quad E_{h_{k, 1}^{m}[n]}\left[1_{\left\{a_{k}[n] \sum_{m} w_{k}^{m}[n] a_{k}[n]=1\right\}}\right] \leq \stackrel{\mathrm{v}}{o} k
$$

We, intend to enable long-term constraint where at each time instant the interference is maintained below a threshold while enabling resource allocation to an optimal level. Here, we consider being the upper bound for those time spans for which interference takes place. To achieve it, we apply the following condition.

$$
E_{h}\left[1_{\left\{a_{k} \sum_{m} w_{k}^{m}(h)=1\right\}}\right] \leq \stackrel{\mathrm{v}}{o} k
$$

The expectation function in (28), considers all CSI realizations. Additionally, the model developed above signifies the joint probability of the PU being active and NC can schedule one SU to transmit over channel. In ISP-DRACM model, to confine the likelihood of one SU to be active while satisfying PU uninterrupted active state, we multiply $\stackrel{\mathrm{v}}{o}$ with the likelihood of the $k$-th radio band to be occupied by the allied PU. Observing (12) and (28), it can be found that the equation (28) still depicts a convex problem (as $E\left[{ }_{h} \sum_{m} w_{k}^{m}(h) 1_{\left\{1_{k}=1\right\}}\right] \leq \stackrel{\mathrm{v}}{o} k$ ). To enable QoS oriented resource allocation in CR-based MANETs, especially under long-term interference, we need to solve it by dualization. To achieve it, we introduce a component $\theta_{k}$ called Lagrange multiplier. Noticeably, in ISP-DRACM, $\theta_{k}$ signifies that the link-quality needs to be redefined as (29).

$$
\varphi_{k}^{m}\left(p_{k}^{m}[n]\right):=\beta^{m} C_{k}^{m}\left(h_{k, 2}^{m}[n], p_{k}^{m}[n]-\pi^{m}[n] p_{k}^{m}[n]-\theta_{k}[n] E_{a_{k}[n]}\left[1_{\left\{a_{k}[n]=1\right\}}\right]\right.
$$


In case of CSI as imperfect, $E_{a_{k}[n]}\left[1_{\left\{a_{k}[n]=1\right\}}\right]=f a_{k}[n]_{2}$, On contrary, for perfectly known CSI, it would be $a_{k}[n]$. Applying the trade-off between transmission rate and power, (29) can penalize SU transmissions which can give rise to the interference whose "price" can be multiplied by the instantaneous probability of interference. Additionally, (29) reveals the role of the primary CSI, secondary CSI and CSI imperfections in resource allocation scheduling. Now, replacing (29) into (14) and (16), we obtain the optimal power for transmission; however there can be the probability of different resource allocation in these cases. Observing the equation it can be found that in short-term interference when $E_{a_{k}[n]}\left[1\left\{a_{k}[n]=1\right\}\right]>\stackrel{v}{o} k$ there is no transmission or resource access scheduled. On contrary, with (29), ISP-DRACM enables transmission even when the interference probability is high by fulfilling the following condition.

$$
\max _{m}\left\{\beta^{m} C_{k}^{m}\left(h_{k, 2}^{m}[n], p_{k}^{m *}[n]-\pi^{m}[n] p_{k}^{m *}[n]\right\}_{m=1}^{M}>\theta_{k}[n] E_{a_{k}[n]}\left[1_{\left\{a_{k}[n]=1\right\}}\right]\right.
$$

Knowing the status that $a_{k}[n]=1$, it enables $\mathrm{SU}$ to access the spectrum provided the $\mathrm{SU}$ possess reward $\theta_{k}[n]$ higher than the cost of interference. In ISP-DRACM, $\theta_{k}[n]$ has been tuned in such manner that the percentage of interfering transmissions remains lower than the predefined limit fixed by $\stackrel{\mathrm{v}}{o} k$. Additionally, $\varphi_{k}^{m}\left(p_{k}^{m}[n]\right)$ too is independent of $p_{k}^{m}[n]$, and thus the similarity in between the transmission power in (14) and the Water Filling Concept" remains realizable and valid. Thus, the decisive disparity in between the short term and the long-term solutions (in interweave CRN set up) primarily vary in the way in which resource scheduling is performed. For short term scenario emphasis is not made towards winner SU-centric rather we focus on giving priority to PU and allied QoS needs. A SU can transmit or access resource only when the interference caused due to SU remains lower than the defined threshold. On contrary, for long-term condition resource scheduling is more flexible and enables opportunistic benefits to SUs without impacting PU concerns.

\section{B. Long-term interference in Underlay CRN}

To enable optimal resource allocation in long-term constraints, we implement the concept of dualization that at first obtains the likelihood of interference to the PU and allied resource allocation. This process, being independent of CSI imperfections makes resource allocation optimization as non-convex. In ISP-DRACM to enable interference resilient resource allocation we formulated our model by confining instantaneous interference probability under underlay setup that gives rise to the following condition (31).

$$
\operatorname{Pr}\left\{\sum_{m} p_{k}^{m}[n] h_{k, 1}^{m}[n]>\Gamma_{k} \mid n\right\} \leq \stackrel{\mathrm{v}}{o} k
$$

In other way, resource scheduling requires fulfilling (32).

$$
E_{h_{k, 1}^{m}[n]}\left|1_{\left\{p_{k}^{m}[n] h_{k, 1}^{m}[n]>\Gamma_{k}\right\}}\right| \leq \stackrel{\mathrm{v}}{o} k
$$

For long-term interference which can be common in case of CRNs, with all channels while hypothesizing SUs to be causing interference, we derive a constraint to be followed (33).

$$
E_{h}\left[\sum_{m} w_{k}^{m}(h) 1\left\{p_{k}^{m}[h] h_{k, 1}^{m}[h]>\Gamma_{k}\right\}\right] \leq \stackrel{\mathrm{v}}{o}
$$

Thus, performing averaged CSI estimation over all $h$ in (33), the resource allocation is performed even without assessing constraints fulfillment over each realizations. Applying (33) in (12), the aforesaid problem turns out to be non-convex and hence becomes complicate to solve. Here we apply averaging over time period that gives rise to the non-convexity problem and hence the result can be leveraged by solving zero duality gap. To be noted, the zero duality gap states that any dual concept can be applied to achieve or relax the interference constraints without compromising the optimality of resource allocation. However, Lagrange remains as non-convex which requires certain robust method to achieve relaxation while enabling optimal resource allocation under constrained interference condition. The following discussion presents the implementation of Lagrange relaxation to achieve constrained resource allocation in CRN.

Consider that the Lagrange relaxation or multiplier allied with certain $k$-th channel be $\vartheta_{k}$ in (33). Now, for interweave CRNs, the use of any new or updated multiplier or relaxation parameter (in Lagrange method) might change link quality which can eventually impact QoS provision. Noticeably, the link quality changed can be obtained as (34).

$$
\varphi_{k}^{m}\left(p_{k}^{m}[n]\right):=\beta^{m} C_{k}^{m}\left(h_{k, 2}^{m}[n], p_{k}^{m}[n]-\pi^{m}[n] p_{k}^{m}[n]-\vartheta_{k}[n] E_{h_{k, 1}^{m}[n]}\left[1_{\left\{p_{k}^{m}[n] h_{k, 1}^{m}[n]>\Gamma_{k}\right\}}\right]\right.
$$


Now considering (29) and (34) the mathematical model derived in (34) use primary as well as secondary CSI in addition to the trades off rate reward with cost of interference and associated transmission power. Replacing (13) with (34), it can be found that the optimal power in (14) (i.e., without interference) and allied transmission (and/or resource) scheduling (16) remain same; however, (26) losses relation with (15) because the third entity in (34) depends on the transmission power, while the optimal power relies merely on initial two terms (15). Practically, due to non-convexity of $\varphi_{k}^{m}\left(p_{k}^{m}[n]\right)$ substituting (34) into (14) is difficult to reward better power efficiency and transmission control. However, improving $\varphi_{k}^{m}\left(p_{k}^{m}[n]\right)$ requires single variable and hence it can be optimized efficiently. Retrieving $\left(p_{k}^{m *}[n]\right\}_{m=1}^{M}$ we need to estimate $\left(w_{k}^{m *}[n]\right\}_{m=1}^{M}$. Noticeably, we need addressing both users as well as spectrum aspects to optimize Lagrange multiplier which has been solved as a one-dimensional non-convex problem. In ISP-DRACM for perfect CSI condition, the power transmission optimization has been performed as follows:

Consider that the maximum power to be transmitted (while ensuring interference resilient transmission and resource access) be $p_{k}^{m^{\prime \prime}}[n]:=h_{k, 1}^{m}[n] / \Gamma_{k}$. Additionally, $\widehat{p}_{k}^{m *}[n]$ be the optimal power (15) (without consider any interference presence). In this case, it fulfills the following condition (35).

$$
p_{k}^{m *}[n]:=\left\{\begin{array}{c}
\hat{p}_{k}^{m *}[n] \text { if }\left(\hat{p}_{k}^{m *}[n]<p_{k}^{m^{\prime \prime}}[n]\right) \vee\left(\begin{array}{c}
\varphi_{k}^{m}\left(\hat{p}_{k}^{m *}[n]\right)> \\
\varphi_{k}^{m}\left(p_{k}^{m^{\prime}}[n]\right)
\end{array}\right) \\
p_{k}^{m^{\prime \prime}}[n] \text { Otherwise }
\end{array}\right.
$$

In ISP-DRACM, in case of high interference cost, the power transmission is confined to a level $p_{k}^{m^{\prime \prime}}[n]$. On contrary, with acceptable level of (low) cost of interference $p_{k}^{m *}[n]$ is permitted to surpass the upper bound. In case of imperfect primary CSI, obtaining $F h_{k, 1}^{m}$ can be helpful to control power optimization. Maintaining $F h_{k, 1}^{m}$ as monotonic, the power optimization problem is always non-convex and with small search space of $F h_{k, 1}^{m}$, the local optima of $\varphi_{k}^{m}\left(p_{k}^{m}[n]\right)$ can be small which can eventually help achieving global optima for efficient resource allocation without imposing interference to the PU. To obtain these suitable parameters and to achieve optimal power transmission control, we have applied Lagrange relaxation method. A snippet of the Lagrange relaxation model is given as follows.

\subsection{Lagrange relaxation}

In our proposed resource allocation strategy we intend to estimate the optimal values for $\pi^{m}[n], \theta_{k}[n]$ and $v_{k}[n]$ so as to achieve optimal power transmission and allied resource allocation. Being the problem of zero duality gap, assigning $\pi^{m}[n]=\pi^{m *}$, $\left.\theta_{k}[n]=\theta^{*}{ }_{k}\right]$ and $v_{k}[n]=v^{*}{ }_{k}$, can also achieve the solution, where $\left(\pi^{m *}, \theta^{*}{ }_{k}, v^{*}{ }_{k}\right\}$ signifies corresponding values for optimizing the dual function associated to (12). Substituting equation (13) into equation (35), the resource allocation can be achieved as optimal for (12). However, this method are highly complex and intricate as there is the need to estimate $\left(\pi^{m *}, \theta^{*}{ }_{k}, v^{*}{ }_{k}\right\}$ iteratively for each time-step and even requires averaging over all possible states of $h$. In addition, such approaches can be limited due to imperfect CSI condition and iteratively estimating the channel statistics $\left(\pi^{m *}, \theta^{*}{ }_{k}, v^{*}{ }_{k}\right\}$ can be too complex and costly. It can cause significant delay in resource allocation which may decisively impact the QoS of MANET. Considering the efficacy of the stochastic prediction based approaches for multiplier estimation in dynamic learning and scheduling purposes, we design a novel and robust model which estimates $\left(\pi^{m *}, \theta^{*}{ }_{k}, v^{*}{ }_{k}\right\}$ and updates it iteratively. We have applied this method as it maintains low computational complexity (which is common in classical methods where authors estimate the optimal values of $\left(\pi^{m *}, \theta^{*}{ }_{k}, v^{*}{ }_{k}\right\}$, which in later phase turns out to be outdated due to network dynamism. Thus, the proposed model estimates the decision parameters dynamically and hence adapts to the dynamic spectrum, which becomes of great significance when PU is very close to the $\mathrm{SU}$ and there is the probability of interference. Thus, obtaining the optimal values of $\mu_{\pi}, \mu_{\theta}, \mu_{v}$, we derive the optimal value of the relaxation coefficient or the multipliers.

$$
\begin{gathered}
\pi^{m}[n+1]=\left[\pi^{m}[n]-\mu_{\pi}\left(\stackrel{\mathrm{v}}{p}-\sum_{k} w_{k}^{m *}[n] p_{k}^{m *}[n]\right]_{0}^{\infty}\right. \\
\theta_{k}[n+1]=\left[\theta_{k}[n]-\mu_{\theta}\left(\stackrel{\mathrm{v}}{o k}-E_{a_{k}[n]}\left[1_{\left(a_{k}[n]=1\right\}} \sum_{m} w_{k}^{m *}[n]\right]\right)\right]_{0}^{\infty} \\
v_{k}[n+1]=\left[v_{k}[n]-\mu_{v}\left(\check{o} k-E_{a_{k}[n]}\left[1_{\left\{a_{k}[n]=1\right\}}\right]\right)\right]_{0}^{\infty} \\
E_{h_{k, 1}^{m}[n]}\left[1_{\left\{p_{k}^{m}[n] h_{k, 1}^{m}[n]>\Gamma_{\mathrm{k}}\right\}} \sum_{m} w_{k}^{m *}[n]\right]_{0}^{\infty}
\end{gathered}
$$

Considering the optimization objectives, the above derived model (36-38) facilitates a fair stochastic sub-gradient of the dual function for the optimal resource allocation (12). In ISP-DRACM resource allocation is scheduled under interference constrained scenario without causing any performance degradation. Thus, obtaining the following values 


$$
\begin{aligned}
& \mu_{m}=\max \left\{\mu_{\pi}, \mu_{\theta}, \mu_{v}\right\} \\
& \bar{p}^{m}:=\frac{1}{n} \sum_{l=1}^{n} \sum w_{k}^{m *}[l] p_{k}^{m *}[l] \\
& \bar{c}^{m}:=\frac{1}{n} \sum_{l=1}^{n} \sum_{k, m} \beta^{m} w_{k}^{m *}[l] C_{k}^{m}\left(h_{k, 2}^{m}[l], p_{k}^{m *}[l]\right) \text { and } \\
& \bar{o}_{k}(n]:=\frac{1}{n} \sum_{l=1}^{n} \sum w_{k}^{m *}[l]\left\{a_{k}[l]=1\right\} \text { (for interweave) and } \\
& \bar{o}_{k}[n]:=\frac{1}{n} \sum_{l=1}^{n} \sum w_{k}^{m *}[l] 1_{p_{k}^{m *}[l] h_{k, 1[l]>\Gamma_{k}}^{m}} \text { (for Underlay)., we ensure the interference resilient resource allocation by fulfilling }
\end{aligned}
$$

conditions given as, $n \rightarrow \infty: \quad 1) . \stackrel{\bar{p}}{p}^{m}[n]=\stackrel{\mathrm{v}}{p}^{m}$ and $\bar{o}_{k}[n]=\bar{o}_{k}$, and 2) $\bar{c}[n] \geq \bar{c}^{*}-\delta(\mu)$, where $\delta(\mu) \rightarrow 0$ as $\mu \rightarrow 0$. The simulation results obtained for the proposed ISP-DRACM resource allocation and power management strategy under different interference setup is discussed in the next section.

\section{Results and Discussion}

This research focused on ensuring resource allocation while maintaining low interference or noise onto the PUs. In our proposed model and allied simulation, realizing network dynamism we introduced varying channel condition at the different instants, where the channel condition and corresponding allocation scheduling was performed over continuous time-series over a definite span. Noticeably, being stochastic prediction based resource allocation and/or power transmission strategy over mobile topology of MANET, performing power transmission control over each time instant $n$ is must and therefore we performed scheduling over $\mathrm{n} . \Delta \mathrm{T}$ time span, which was considered as $10000 . \mathrm{n} . \Delta \mathrm{T}$ can also be stated as the total simulated time instant over which the resource scheduling was performed. In our proposed ISP-DRACM model SUs were assigned with the initial transmission power, and the total number of SUs considered was 10, while only two nodes were assigned as PU. Some of the key simulation parameters used in ISP-DRACM is given in Table 1 .

Table 1. Simulation environment

\begin{tabular}{ll}
\hline Parameter & Value \\
\hline Network type & CRN \\
Number of users & 8 \\
Number of frequency bands & 10 \\
Average transmit power constraint per CR & $P_{\text {avg }}=$ ones(No. of users, 1$)$ \\
Maximum Interference probability from SU onto PU & $.04^{*}$ ones(No. of frequency bands,1) \\
SNR for which interference occurs & $.5^{\star}$ ones(No. of frequency bands,1) \\
Average Power allowed at Prim. Rx Side (for comp. purposes) & $.5^{*}$ ones(No. of frequency bands, 1) \\
User priority coefficient & ones(No. of users,1) \\
Average SNR of the secondary channel & $9^{*}$ ones(No. of users, No. of frequency bands) \\
SNR gap of the modulation w.r.t. Shannon's limit & $1^{\star}$ ones(No. of users, No. of frequency bands) \\
Activity Sensing duration & $3 \mathrm{sec}$ \\
Time correlation coefficient & 0.95 \\
Inverse noise & $(1-$ Time correlation coefficient) \\
Error in the analog measurement & 0.01 \\
Estimation interval of the primary channel & $6 \mathrm{sec}$ \\
Number of simulated time instants & $10000 \mathrm{sec}$ \\
\hline
\end{tabular}

To assess robustness of the proposed ISP-DRACM resource allocation model, we simulated it for the different interference conditions or constraints such as interweave setup, underlay setup with both short-term as well as long-term interference constraints. We simulated ISP-DRACM under both known CSI as well as unknown CSI conditions; though in this simulation results we have discussed the outcome for known CSI only. To adopt realistic noise conditions in MANET systems, we considered the amplitudes of the SU channels as Rayleigh and distributed for which we maintained real and imaginary components independent. On the other hand, the primary CSI was considered to be Gaussian distributed with mean as zero and unit variance. We considered time correlated model as $H_{k, 1}^{m}[n]=\sqrt{\gamma} H_{k, 1}^{m}[n-1]+\sqrt{1-\gamma A_{k}^{m}}$ where $\gamma=0.98$ and $A_{k}^{m}$ is white and distributed in exponential manner. The deployed network controller model senses SUs every 3 second, while PUs at the interval of 6 seconds. In ISP-DRACM we considered likelihood of false alarm as 3\%, while the likelihood for miss detection 
was limited to merely $2 \%$.

To examine performance of the proposed ISP-DRACM resource allocation model, we assessed its efficacy in terms of power transmission, interference, channel utilization etc. As stated, to achieve optimal resource allocation we tried to maintain high weighted sum rate of the secondary users by maintaining interference below a defined threshold. To achieve it, we solved (6) by applying RBEM-HMM with Lagrange relaxation, where it predicted interference stochastically with reference to which the Lagrange multipliers were obtained to reduce interference. The simulations were performed for instantaneous as well as average interference conditions over underlay and interweave (overlay) CRN setup (for CR-based MANET). To illustrate dynamic performance by ISP-DRACM, we have plotted evolution of the noise, interference, Lagrange relaxation variables etc. over simulation period. Some of the key performance assessment variables are discussed as follows. Noticeably, being based on duality solving problem, we have obtained simulation results in the form of trajectory evolution for the different primal and dual variables which are plotted against the standard performance with known optimal values. Due to the space constraints in this manuscript, we have examined parametric evolution and respective resource allocation performances under known CSI condition for both interweave as well as underlay network.

The parametric evolution and respective interference condition over interweave CR setup is given in Figures 1, 2 and 3. Noticeably, the simulation results obtained are simulated with ISP-DRACM over five channels (i.e., $\mathrm{k}=5$ ). Figures 1, 2 and 3 presents the evolution or trajectory of primal and dual variables for CR setup with known CSI condition.

In Figure 1, the first subplot states the instantaneous power of the detected signal from the perfectly known CSI, which the corresponding sample average power is depicted in Figure 1(b). Figure 1(c) presents the trajectory of the primal and dual variables of instantaneous power multipliers, and as it can be observed here, the proposed model achieves convergence just within 600 iterations. It exhibits robustness of the proposed HMM-Lagrange relaxation model to achieve convergence soon while reducing interference to zero on PUs. In the proposed stochastic resource allocation strategy, the formulation was made in such manner that ISP-DRACM maximizes the weighted sum-rate of orthogonally transmitting SUs under sample average-power and probabilistic interference constraints. It can be well visualized through the results. Observing the result it can be found that the proposed method with stochastic resource allocation ability achieves zero-duality. Results depict the instantaneous values for the Lagrange relaxation for power transmission control and interference suppression, respectively. 
(a) Instantaneous Powers

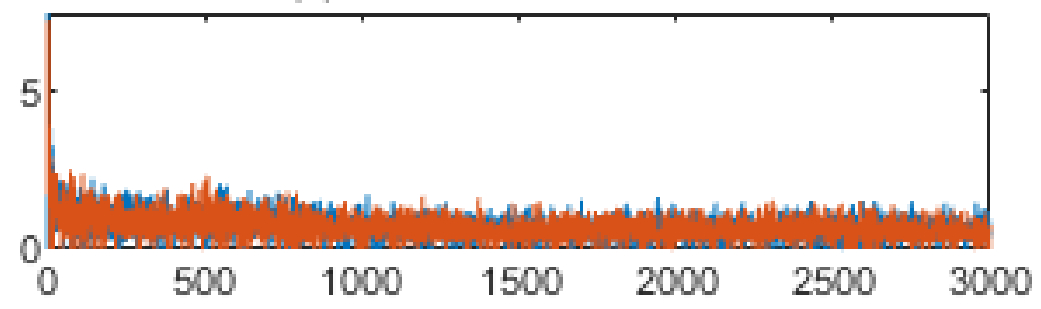

(b) Sample Average Powers

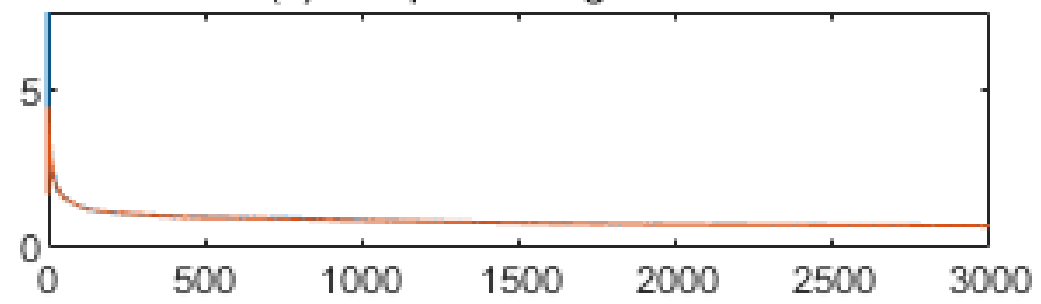

(c) Instantaneous Power Multipliers

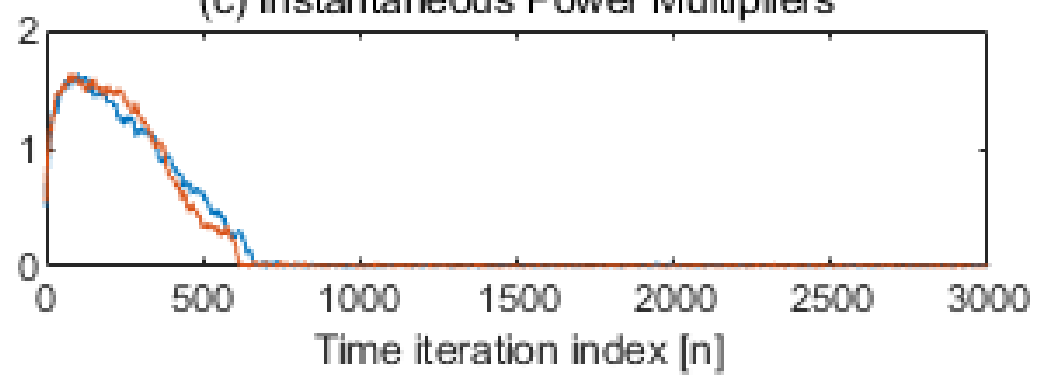

Fig 1. Signal sensing and corresponding primal and dual parametric evolution with 10 SU and 5 channels (known CSI using NC). (a) Instantaneous powers, (b) sample average power and (c) instantaneous power multipliers (Lagrange). 
(a) Instantaneous Interfering Powers

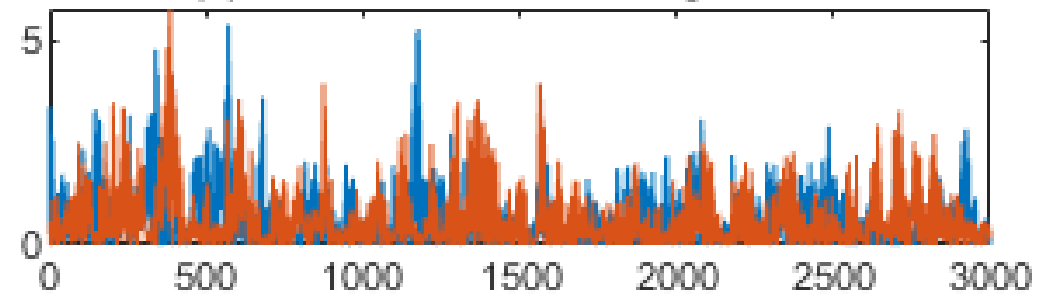

(b) Sample Average Interfering Powers

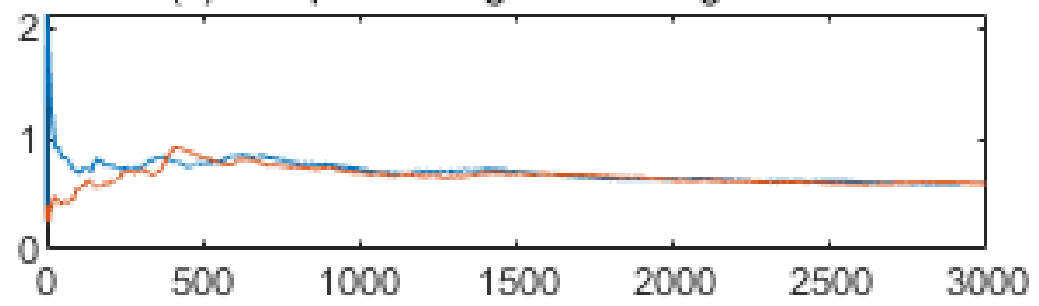

(c) Instantaneous Interfering Power Multipliers

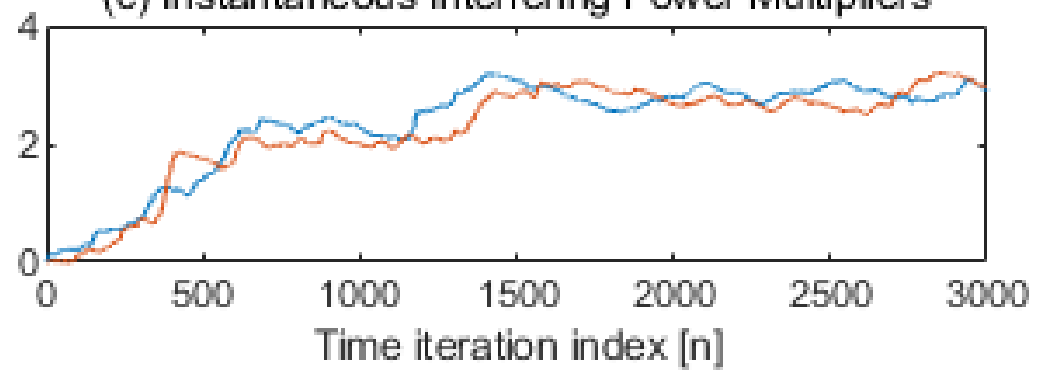

Fig 2. Evolution of the different primal and dual variables with known CSI. (a)Instantaneous interfering powers over different channel, (b)Sample average interference power and (c)Instantaneous power (Lagrange) multipliers over varying time iteration index [n].

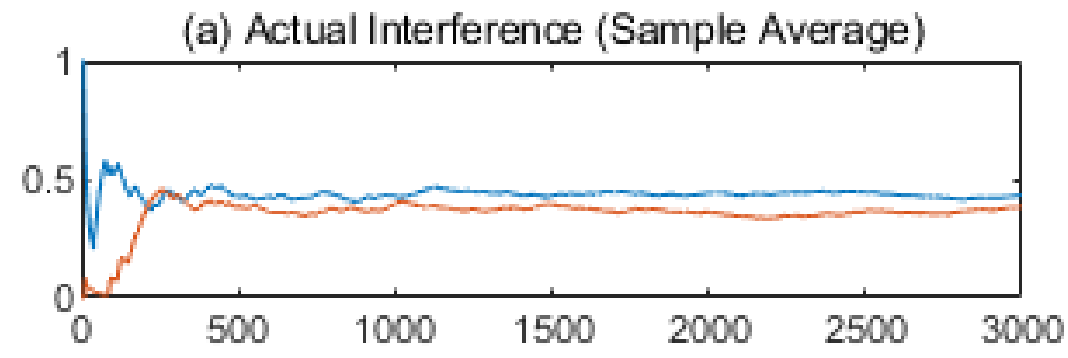

(b) Estimated Interference (Sample Average)

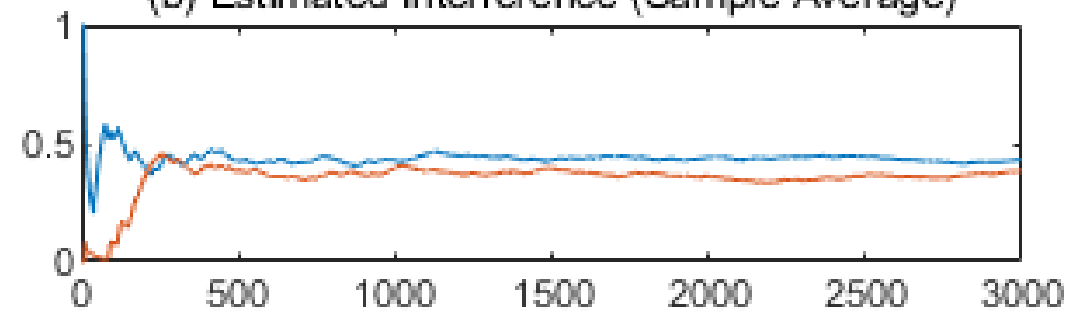

Fig 3. Evolution of the different primal and dual variables with known CSI. (a)actual interference, (b) estimated interference power with sample average ( $\mathrm{x}$-axis states time iteration index $[\mathrm{n}]$ 
Observing overall performance it can be visualized that the proposed model achieves convergence just after a few hundreds of the iterations and achieves stated constraints successfully to enable dynamic resource allocation to the users without imposing interference to the PUs in CRN. The resource allocation performance too affirms that the proposed model achieves optimal resource allocation while maintaining efficient transmission scheduling for SUs. The proposed model and allied simulation outputs substantiate the efficacy of the proposed resource allocation model under different CRN conditions. The proposed model fulfils expected constraints while indicating that avoiding CSI perfection might cause sub-optimal solution for resource allocation. Our proposed model affirms that exploiting statistical information and allied CSI/Interference over SU-to-PU channels can help making optimal resource allocation decision. This study confirmed that the resource allocation average or the long-term interference constraints yields slightly better resource allocation, as compared to the instantaneous one; however yields satisfactory performance in terms of noise resilience. Additionally, the interference probability estimation by our proposed stochastic prediction model helped updating belief states as per the real channel and node condition that avoided further collision and QoS violation. The resource allocation performance by the proposed ISP-DRACM model with known CSI information is depicted in Figure 4. Considering buffer occupancy performance Figure 4 depicts that the proposed system intends to use resources maximum possible while maintaining minimum interference on PU(s). Noticeably, the proposed method has exhibited satisfactory performance towards spectrum allocation to the SU without imposing any significant interference for both interweave as well as underlay CR setup. Additionally, the robustness of the proposed ISP-DRACM model over varying CSI and associated network parameters enable it to be compatible for MANETs, where it can work as a middleware to perform optimal QoS-centric resource management.

(a) Buffer Occupency

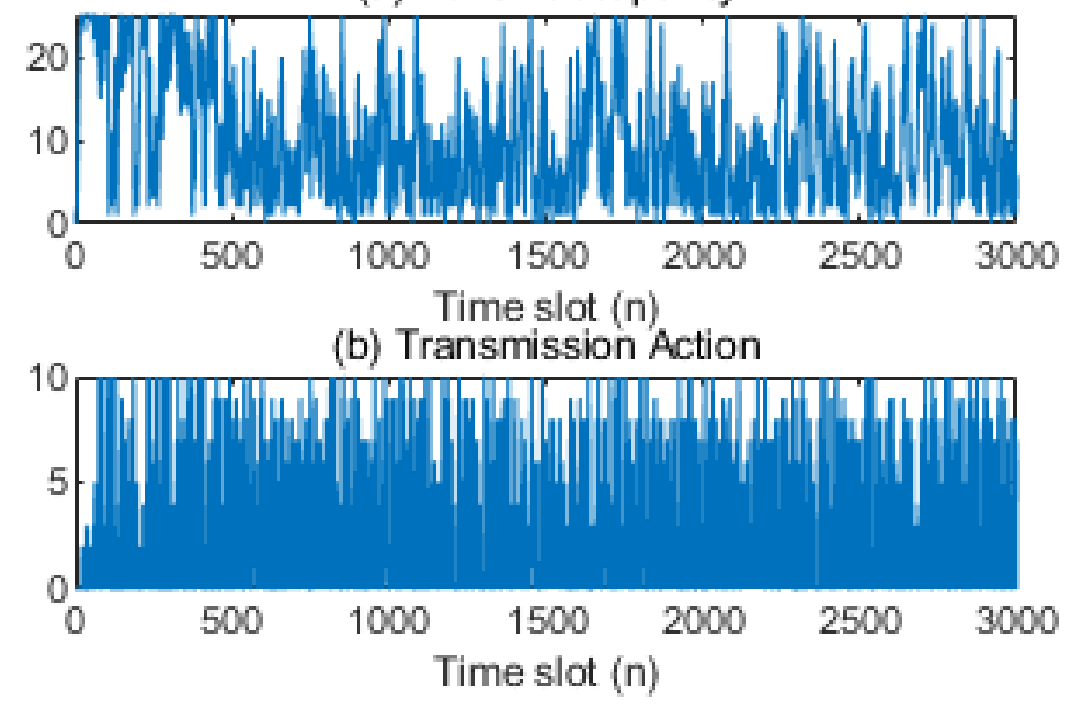

Fig 4. Transmission control and corresponding buffer occupancy over n samples.

\section{Conclusion}

In this study, the predominant emphasis was made on designing a robust interference resilient dynamic resource allocation strategy for CR-based MANETs. Realizing the dynamic topology and opportunistic resource demand nature of CR-based MANET which can be of both interweave and underlay types, the proposed model intended to maintain high weight sum rate for SUs while maintaining interference and noise lower than a defined threshold. Here, the prime objective was to maintain interference and noise component caused due to SUs lower than an acceptable level while ensuring optimal source allocation to the users, under interweave and underlay conditions. As interference constraints, the proposed model considered both instantaneous as well as average interference constraints for which the optimal resource allocation was performed. In the proposed model, the dynamic resource allocation was scheduled as a Markov Decision Problem (MDP), where it intended to maintain or provide maximum resource utilization while limiting interference caused by SUs onto the PUs. As stated, to achieve stochastic resource allocation the proposed method intended to achieve sum-rate maximization while constraining maximum average power and interference probability. Considering network dynamism the probabilistic interference was modeled in such 
manner that it depicted errors and imperfections in spectrum sensing and CSI estimation. The consideration of both short-term as well as long term interference and allied dynamic resource allocation exhibits robustness of the proposed model. For short term interference the proposed model considered CSI imperfections to ensure that the interference probability at any instant remains lower than a defined level. On the other hand, the long term interference exploited the differences of the interference over different time period to ensure that the time-span during which interference occurs doesn't exceed a defined level or threshold. The proposed model considered above stated problem as non-convex problem which was solved as zero-duality gap problem. Thus, maximizing the rate (signifying the quality of secondary links), transmission power and optimizing interference with reference to the PUs, the proposed model achieved optimal resource allocation. To achieve swift convergence, Lagrange multiplier or relaxation parameters were multiplied with above stated parameters, where Lagrange multiplier value itself was obtained with reference to the demands of the PUs and SUs in CR-based MANETs. Summarily, the use of stochastic model helped achieving probability of interference and optimal values of the Lagrange multipliers which helped enabling optimal resource allocation to the users across CRNs.

\section{References}

1) Liang Z, Feng S, Zhao D, Shen X. Delay performance analysis for supporting real-time traffic in a cognitive radio sensor network. IEEE Transactions on Wireless Communications. 2011;10(1):325-335. Available from: https://doi.org/10.1109/TWC.2010.111910.100804.

2) Bicen AO, Gungor VC, Akan OB. Delay-sensitive and multimedia communication in cognitive radio sensor networks. Ad Hoc Networks. 2012;10(5):816830. Available from: https://dx.doi.org/10.1016/j.adhoc.2011.01.021.

3) Lin S, Chen K. Improving spectrum efficiency via in-network computations in cognitive radio sensor networks. IEEE Transactions on Wireless Communications. 2014;13(3):1222-1234. Available from: https://doi.org/10.1109/TWC.2014.011514.121905.

4) Spachos P, Hantzinakos D. Scalable Dynamic Routing Protocol for Cognitive Radio Sensor Networks. IEEE Sensors Journal. 2014;14(7):2257-2266. Available from: https://dx.doi.org/10.1109/jsen.2014.2309138.

5) Helal E, Khattab A, Fahmy YA. Energy-efficient cluster head selection for cognitive radio sensor networks. In: proceedings of 28 th International Conference on Microelectronics (ICM). 2016;p. 205-208.

6) Mesodiakaki A, Adelantado F, Alonso L, Verikoukis C. Energy-efficient user association in cognitive heterogeneous networks. IEEE Communications Magazine. 2014;52(7):22-29. Available from: https://dx.doi.org/10.1109/mcom.2014.6852079.

7) Imtiaz J, Kim D. Energy-Efficient Management of Cognitive Radio Terminals With Quality-Based Activation. IEEE Communications Letters. 2017;21(5):1171-1174. Available from: https://dx.doi.org/10.1109/lcomm.2017.2656910.

8) Chatziantoniou E, Allen B, Velisavljevic V. An HMM-based spectrum occupancy predictor for energy efficient cognitive radio. In: IEEE 24th Annual International Symposium on Personal, Indoor, and Mobile Radio Communications (PIMRC). 2013;p. 601-605.

9) Oloyede A, Grace D. Energy efficient learning based auction process for cognitive radio systems. In: proceedings of IEEE 11th Consumer Communications and Networking Conference. 2014;p. 35-40.

10) Kaschel H, Toledo K. Energy efficient spectrum management in cognitive radio sensor networks. In: CHILEAN Conference on Electrical, Electronics Engineering, Information and Communication Technologies. 2017;p. 1-8.

11) Chatterjee S, Maity SP, Acharya T. Energy Efficient Cognitive Radio System for Joint Spectrum Sensing and Data Transmission. IEEE Journal on Emerging and Selected Topics in Circuits and Systems. 2014;4(3):292-300. Available from: https://dx.doi.org/10.1109/jetcas.2014.2337191.

12) Chen J, Kuo Y, Liu Y, Lv L, Ren C. Energy efficient relay selection and power allocation for cooperative cognitive radio networks. IET Communications. 2015;9(13):1661-1668. Available from: https://dx.doi.org/10.1049/iet-com.2014.1246.

13) Liu B, Lu G, Li Z, Zhou F. Spectrum sensing interval optimization and power control for energy efficient cognitive radio networks. In: Proceedings of 2017 9th International Conference on Wireless Communications and Signal Processing (WCSP). 2017;p. 1-6.

14) Das D, Das S. A novel resource allocation for energy-efficient Cognitive Radio. In: Proceedings of 2015 Annual IEEE India Conference (INDICON). 2015;p. 1-6.

15) Shi Z, Li KH, Tan T, Teh KC. Energy efficient cognitive radio network based on multiband sensing and spectrum sharing. IET Communications. 2014;8(9):1499-1507. Available from: https://dx.doi.org/10.1049/iet-com.2013.0699.

16) Li A, Han G, Ohtsuki T. Energy-Efficient Channel Hopping Protocol for Cognitive Radio Networks. In: Proceedings of GLOBECOM 2017 - 2017 IEEE Global Communications Conference. ;p. 1-6.

17) Alabbasi A, Rezki Z, Shihada B. Energy efficiency and SINR maximization beamformers for cognitive radio utilizing sensing information. In: IEEE International Symposium on Information Theory. 2014;p. 1391-1395.

18) Haddad M, Hayel Y, Habachi O. Spectrum Coordination in Energy-Efficient Cognitive Radio Networks. IEEE Transactions on Vehicular Technology. 2015;64(5):2112-2122. Available from: https://dx.doi.org/10.1109/tvt.2014.2339271.

19) Li Y, Zh R, Lu Y. Smart relay opportunistic spectrum sharing in energy efficient cognitive radio networks, proceedings of. In: IEEE International Conference on Communication Systems. 2014;p. 283-287.

20) Yang J, Zhao H, Chen X, Xu J, Zhang J. Energy-efficient design of spectrum prediction in cognitive radio networks: Prediction strategy and communication environment. In: proceedings of 12th International Conference on Signal Processing. 2014;p. 154-158.

21) Sboui L, Ghazzai H, Rezki Z, Alouini M. Energy-Efficient Power Allocation for UAV Cognitive Radio Systems. In: IEEE 86th Vehicular Technology Conference (VTC-Fall). 2017;p. 1-5.

22) Yadav R, Singh K, Kumar A, Kumar T. Energy-efficient power allocation with spectrum sensing in cognitive radio networks. In: IEEE/CIC International Conference on Communications in China (ICCC). 2015;p. 1-6.

23) Kamruzzaman SM, Hossain MA, Alghamdi A. Energy efficient cognitive radio MAC protocol for battlefield communications. In: proceedings of 2015 IEEE 28th Canadian Conference on Electrical and Computer Engineering (CCECE). 2015;p. 1101-1108.

24) Moualeu JM, Ngatched TMN, Hamouda W, Takawira F. Energy-efficient cooperative spectrum sensing and transmission in multi-channel cognitive radio networks. In: proceedings of IEEE International Conference on Communications (ICC). 2014;p. 4945-4950. 
25) Kozal SB, Merabti M, Bouhafs F. Energy-efficient multi-hop clustering scheme for cooperative spectrum sensing in cognitive radio networks. In: proceedings of 2014 IEEE 11th Consumer Communications and Networking Conference (CCNC). 2014;p. 139-145.

26) Shirke N, Patil K, Kulkarni S, Markande S. Energy efficient cluster based routing protocol for distributed Cognitive radio network. In: proceedings of First International Conference on Networks and Soft Computing. 2014;p. 60-65.

27) Marques AG, Dall'Anese E, Giannakis GB. Cross-Layer Optimization and Receiver Localization for Cognitive Networks Using Interference Tweets. IEEE Journal on Selected Areas in Communications. 2014;32(3):641-653. Available from: https://dx.doi.org/10.1109/jsac.2014.1403009.

28) Lopez-Ramos LM, Marques AG, Ramos J. Soft-decision sequential sensing for optimization of interweave Cognitive Radio networks. In: IEEE 14th Workshop on Signal Processing Advances in Wireless Communications (SPAWC). 2013;p. 235-239.

29) Saki H, Shikh-Bahaei M. Cross-Layer Resource Allocation for Video Streaming Over OFDMA Cognitive Radio Networks. IEEE Transactions on Multimedia. 2015;17(3):333-345. Available from: https://dx.doi.org/10.1109/tmm.2015.2389032.

30) Mokari N, Azmi P, Saeedi H. Quantized Ergodic Radio Resource Allocation in Cognitive Networks with Guaranteed Quality of Service for Primary Network. IEEE Transactions on Vehicular Technology. 2014;99(8). Available from: https://doi.org/10.1109/TVT.2014.2306133. 